\title{
Random walk on finite extensions of lattices
}

\section{Marche aléatoire sur les extensions finies de réseaux}

\section{Vignon Oussa}

Department of Mathematics, Bridgewater State University, Bridgewater, MA 02324, U.S.A.

vignon.oussa@bridgew.edu

ABSTRACT. We obtain a precise formula for the probability that a random walker returns to the origin after $n$ steps on some semi-direct product groups obtained by extending a Euclidean lattice by a finite group. Prior to this work, to the best of our knowledge, for the class of groups considered, only asymptotic estimates were available in the literature.

2010 Mathematics Subject Classification. 60B15,43A30,43A40

KEYWORDS. random walk,semidirect groups, finite extension.

\section{Introduction and overview of the work}

According to the well-known Pòlya's recurrence theorem, a simple random walk on a $d$-dimensional lattice is recurrent when $d=1$ or $d=2$ and transient otherwise. Intuitively, the results of Pòlya can be understood as follows [7]. We regard the lattice $\mathbb{Z}^{d}$ as a graph whose vertices are points with coordinates in the set of integers, and an edge joins two points if the distance between them is equal to one. A random walker departs from the origin and chooses its next destination among the $2 d$ neighboring points of its current location with equal probability. As the set of minimal generators of $\mathbb{Z}^{d}$ increases in size, the total number of available routes to the walker will go up. In other words, as $d$ gets larger, it gets harder for a random walker to return to its point of departure.

The probability that a random walker on $\mathbb{Z}^{d}$ will return to its initial position after $k$ many steps is zero if $k$ is odd. Otherwise, this probability is given by the total number of closed paths of length $k$ starting at the identity over the quantity $(2 d)^{k}$. Note that even without appealing to any combinatorial tools, it is still possible to explicitly compute a formula for the probability that a random walker on $\mathbb{Z}^{d}$ will return to its initial position after a prescribed number of steps. Indeed, letting $p$ be a probability distribution on $\mathbb{Z}^{d}=\mathbb{Z}$-span $\left\{e_{k}: 1 \leq k \leq d\right\}$ such that

$$
p(x)=\left\{\begin{array}{c}
\frac{1}{2 d} \text { if } x \in\left\{ \pm e_{k}: 1 \leq k \leq d\right\} \\
0 \text { otherwise }
\end{array},\right.
$$

the $n^{\text {th }}$ convolution power $p^{(n)}=p * \cdots * p$ is the distribution of the associated random walk at the $n^{\text {th }}$ step. In other words, the probability of returning at the origin is obtained by evaluating $p^{(n)}$ at the identity element in $\mathbb{Z}^{d}$. Letting $\mathcal{F}$ be the Fourier transform defined on $l^{1}\left(\mathbb{Z}^{d}\right)$, since $\mathcal{F}$ takes convolution into point-wise multiplication, it is clear that

$$
p^{(2 n)}(0)=\int_{[0,1)^{d}}(\mathcal{F} p(\lambda))^{2 n} d \lambda=\frac{1}{d^{2 n}}\left(\int_{[0,1]^{d}}\left(\cos \left(2 \pi \lambda_{1}\right)+\cdots+\cos \left(2 \pi \lambda_{d}\right)\right)^{2 n} d \lambda\right) .
$$


For instance, for the specific case where $d=1$, since $\left(\begin{array}{c}2 n \\ n\end{array}\right)=\frac{(2 n) !}{(n) !(n) !}$, we quickly recover

$$
\begin{aligned}
p^{(2 n)}(0) & =\int_{[0,1]} \cos ^{2 n}(2 \pi t) d t=\left(\frac{4}{4 \sqrt{\pi}(n) !}\right) \cdot\left(\frac{(2 n) !}{4^{n}(n) !} \sqrt{\pi}\right) \\
& =\frac{(2 n) !}{((n) !)^{2}} \frac{1}{4^{n}}=\left(\begin{array}{c}
2 n \\
n
\end{array}\right) \frac{1}{4^{n}} .
\end{aligned}
$$

The main objective of the present paper is to investigate the extent to which it is possible to generalize Formula (1.1) to non-commutative groups of the type $\mathbb{Z}^{d} \rtimes H$ where $H$ is finite.

\subsection{Short summary of our results}

Let $G=A \rtimes H$ where $A=\mathbb{Z}$-span $\left\{e_{k}: 1 \leq k \leq d\right\}$ is a full rank lattice of $\mathbb{R}^{d}=\mathbb{R}$-span $\left\{e_{k}: 1 \leq\right.$ $k \leq d\}$ and $H=\left\{h_{1}, \cdots, h_{r}\right\}^{1}$ is a finite subgroup of $G L(A)$ [5]. The group $G$ is equipped with the following operation:

$$
(x, z)\left(x^{\prime}, z^{\prime}\right)=\left(x+z x^{\prime}, z z^{\prime}\right) \text { where }(x, z),\left(x^{\prime}, z^{\prime}\right) \in A \times H .
$$

As a starting point, let

$$
K=\left\{\left( \pm e_{1}, \operatorname{id}_{H}\right), \cdots,\left( \pm e_{d}, \operatorname{id}_{H}\right),\left(0, h_{i_{1}}\right), \cdots,\left(0, h_{i_{m}}\right)\right\}
$$

be a minimal symmetric generating set for $G$ and define a sequence $a_{K}$ of finite support on $G$ such that

$$
a_{K}(x)=\left\{\begin{array}{cc}
\frac{1}{|K|} & \text { if } x \in K \\
0 & \text { if } x \notin K
\end{array} .\right.
$$

As such, $a_{K}$ is a positive function satisfying $\left\|a_{K}\right\|_{l^{1}(G)}=1$. A random walk process starting at the identity $\left(0, \mathrm{id}_{H}\right)$ is described by repeatedly selecting points in $K$ with probability $\frac{1}{|K|}$ and multiplying these elements together. We denote by $a_{K}^{(n)}$, the $n$-fold convolution of $a_{K}$ with itself where

$$
a_{K} * a_{K}(u)=\sum_{v \in G} a_{K}(v) a_{K}\left(v^{-1} u\right) \text { and } a_{K}^{(n)}=a_{K} * \cdots * a_{K} .
$$

Appealing to the theory of Mackey [2], we are able to obtain a precise Plancherel measure and an explicit description of the unitary irreducible representations of $G$. This gives us full access to the group Fourier transform of $G$ and its inverse. With these tools at our disposal, we are in a position to investigate the extent to which we can compute the exact probability (with respect to the given distribution) that a random walker on $G$ returns to the identity element in $G$ after $2 n$ steps.

For fixed $\lambda \in \mathbb{R}^{d}$, we define matrices $D(\lambda)$ and $S$ such that

$$
D(\lambda)=\left[\begin{array}{ccc}
2 \sum_{k=1}^{d} \cos \left(2 \pi\left\langle\lambda, h_{1}^{-1} e_{k}\right\rangle\right) & \\
& \ddots & \\
& & 2 \sum_{k=1}^{d} \cos \left(2 \pi\left\langle\lambda, h_{|H|}^{-1} e_{k}\right\rangle\right)
\end{array}\right]
$$

1. $r$ is the order of the finite group $H$ 
and

$$
S=\sum_{k=1}^{m} L\left(h_{k}\right)
$$

where $L$ is the left regular representation of $H$. By assumption $D(\lambda), S$ are square matrices of order $|H|=r$. As we will see in the subsequent section, (see Formula (2.9)) the probability that a random walker will return at the identity after $2 n$ steps is given by means of integration as

$$
a_{K}^{(2 n)}\left(0, \operatorname{id}_{H}\right)=\left(|H| \cdot(2 d+m)^{2 n}\right)^{-1} \cdot \int_{[0,1]^{d}} \operatorname{Tr}\left((D(\lambda)+S)^{2 n}\right) d \lambda .
$$

Note that in the case where $H$ is the trivial group, $m=0, A \rtimes H$ is isomorphic to $A=\mathbb{Z}^{d}$ and $K$ becomes $\left\{ \pm e_{1}, \cdots, \pm e_{d}\right\}$. Moreover, letting $\mathcal{F}$ be the Fourier transform on $l^{2}(\mathbb{Z})$,

$$
\begin{aligned}
{\left[\mathcal{F} a_{K}^{(2 n)}\right](\lambda) } & =\left(\left[\mathcal{F} a_{K}\right](\lambda)\right)^{2 n} \\
& =\left(\sum_{\gamma \in \mathbb{Z}^{d}} a_{K}(\gamma) e^{-2 \pi i\langle\gamma, \lambda\rangle}\right)^{2 n} \\
& =\left(\sum_{\gamma \in\left\{ \pm e_{1}, \cdots, \pm e_{d}\right\}} \frac{e^{-2 \pi i\langle\gamma, \lambda\rangle}}{2 d}\right)^{2 n} \\
& =\left(\frac{1}{d^{2 n}}\right) \cdot\left(\sum_{k=1}^{d} \cos \left(2 \pi \lambda_{k}\right)\right)^{2 n}
\end{aligned}
$$

and

$$
\begin{aligned}
a_{K}^{(2 n)}(0) & =\mathcal{F}^{-1}\left(\mathcal{F} a_{K}^{(2 n)}\right)(0)=\frac{1}{d^{2 n}} \int_{[0,1]^{d}}\left(\sum_{k=1}^{d} \cos \left(2 \pi \lambda_{k}\right)\right)^{2 n} d \lambda \\
& =d^{-2 n} \int_{[0,1]^{d}}\left(\cos \left(2 \pi \lambda_{1}\right)+\cdots+\cos \left(2 \pi \lambda_{d}\right)\right)^{2 n} d \lambda .
\end{aligned}
$$

This allows us to recover Formula (1.1) (see also [7])

To derive an explicit formula for (1.4), we first need to compute the trace of the matrix $(D(\lambda)+S)^{2 n}$ for each natural number $n$ and $\lambda \in[0,1]^{d}$. This is generally a difficult task. Even under the assumption that we know what the eigenvalues of $D(\lambda)$ and $S$ are, the eigenvalues of the operator $D(\lambda)+S$ can still be challenging to compute explicitly.

Our main result gives sufficient conditions under which this can be achieved.

Theorem 1. Let $n$ be a natural number. If $D(\lambda)$ and $S$ (as defined in (1.2) and (1.3) respectively) are simultaneously triangularizable for almost every $\lambda$ with respect to the Lebesgue measure in $[0,1]^{d}$ then there exists a sequence $\left(\omega_{s}\right)_{s=1}^{|H|}$ of eigenvalues for $S$ such that

$$
a_{K}^{(2 n)}\left(0, \operatorname{id}_{H}\right)=\frac{1}{|H|} \sum_{s=1}^{|H|} \int_{[0,1]^{d}}\left(\left(\sum_{k=1}^{d} \frac{2 \cos \left(2 \pi\left\langle\lambda, h_{s}^{-1} e_{k}\right\rangle\right)}{|K|}\right)+\frac{\omega_{s}}{|K|}\right)^{2 n} d \lambda
$$


Corollary 2. If $D(\lambda)$ as defined in (1.2) is a constant multiple of the identity (for almost every $\lambda$ with respect to the Lebesgue measure) then there exists a sequence $\left(\omega_{s}\right)_{s=1}^{|H|}$ of eigenvalues for $S=\sum_{k=1}^{m} L\left(h_{i_{k}}\right)$ such that

$$
a_{K}^{(2 n)}\left(0, \mathrm{id}_{H}\right)=\frac{1}{|H| \cdot|K|^{2 n}} \sum_{s=1}^{|H|} \sum_{\ell=0}^{n} \sum_{\kappa_{1}+\ldots+\kappa_{d}=\ell} \frac{(2 n) !\left(\omega_{s}\right)^{2(n-\ell)}}{(2(n-\ell)) !\left(\kappa_{1} ! \cdots\left(\kappa_{d}\right) !\right)^{2}}
$$

The reader interested in learning more about the connections between random walks on groups and topics such as solid-state physics and ergodic theory is referred to [1].

While the literature contains a wealth of asymptotic estimates for quite general classes of groups (see, for instance, [7]), the main novelty of our results lies in its explicit nature and the approach undertaken in substantiating these findings.

\section{Proof of Theorem 1}

\subsection{Notation and preliminaries}

- The inverse-transpose of a matrix $M$ is denoted by $M^{-1 T}$.

- Let $T$ be a linear operator acting on a Hilbert space $H$ with orthonormal basis $\left(\mathbf{b}_{k}\right)_{k \in I}$

- The adjoint of $T$ is denoted $T^{\star}$

- The trace of $T$ is given by $\operatorname{Tr}(T)=\sum_{k \in I}\left\langle T \mathbf{b}_{k}, \mathbf{b}_{k}\right\rangle$.

- The Hilbert Schmidt norm of a linear operator $T$ is given by $\|T\|_{\mathrm{HS}}^{2}=\operatorname{Tr}\left(T^{\star} T\right)$.

- Suppose that the unitary representation $\pi$ is modeled as acting on a finite-dimensional Hilbert space which we denote by $\mathbf{H}_{\pi}$. The algebra of all bounded linear operators on $\mathbf{H}_{\pi}$ is denoted $\mathcal{B}\left(\mathbf{H}_{\pi}\right)$. Assuming that $\mathbf{H}_{\pi}$ is finite-dimensional, the Hilbert-Schmidt norm which is induced by the inner product $\langle A, B\rangle_{\mathrm{HS}}=\operatorname{Tr}\left(A B^{\star}\right)=\operatorname{Tr}\left(B^{\star} A\right)$ where $A, B \in \mathbf{H}_{\pi}$ and $\|A\|_{\mathrm{HS}}=\left(\operatorname{Tr}\left(A^{\star} A\right)\right)^{1 / 2}$.

\section{A short description of Mackey's analysis}

To obtain a precise formula for the group Fourier transform of $G$, we must first compute its unitary dual and the corresponding Plancherel's measure. To this end, we shall appeal to Mackey's analysis, which provides us with a set of procedures used to describe the unitary dual of $G$ provided that certain regularity conditions [3] are in place, and we also have information about the unitary dual of some normal subgroup $A$ of $G$.

For instance, Mackey's theory is handy for almost abelian groups (groups containing an abelian normal subgroup $\mathrm{N}$ of finite index.) Referring to [3], the process of describing the unitary dual for almost abelian groups can be summarized as follows.

Suppose $A$ is a normal closed subgroup of $G$. For any $\xi$ in the unitary dual of $A$, we let $G_{\xi}=$ $\{x \in G: x \star \xi=\xi\}$ where $x \star \xi(n)=\xi\left(x^{-1} n x\right)$ for $n \in A$. The action of $G$ on the unitary dual of $A$ described above is induced by the conjugation action of $G$ on its normal subgroup $A$. For a fixed unitary representation $\xi$ in the unitary dual of $A, G \cdot \xi=\{x \cdot \xi: x \in G\}$ is the orbit of $\xi$ under this action and $G_{\xi}$ is the corresponding stabilizer. 
Let $\Sigma$ be a subset of the unitary dual of $A$ such that the intersection of $\Sigma$ with each $G$-orbit in the unitary dual of $A$ is a singleton. For each $\lambda \in \Sigma$, let $\widehat{G}_{\lambda}$ denote the set of equivalence classes of irreducible representations of $G_{\lambda}$ which restrict to a multiple of $\lambda$ on $A$. Then the unitary dual of $G$ is the fiber set

$$
\widehat{G}=\bigcup_{\lambda \in \Sigma}\left\{\operatorname{ind}_{G_{\lambda}}^{G}(\pi): \pi \in \widehat{G}_{\lambda}\right\}
$$

having $\Sigma$ as base space (see Theorem 4.3, [3].)

For the class of almost abelian groups, it is known that there exists a Plancherel measure $d \mu$ on $\widehat{G}$ with respect to which Fourier inversion is defined [2]. In a formal sense, for any natural number $n$, and for suitable $a$,

$$
a^{(n)}(e)=\int_{\widehat{G}} \operatorname{Tr}\left((\widehat{a}(\tau))^{n}\right) d \mu(\tau) .
$$

Of course, (2.1) has limited value unless we have at our disposal a precise description of the Plancherel measure $d \mu$ together with an explicit realization of $\operatorname{ind}_{G_{\lambda}}^{G}(\pi)$ for $\lambda \in \Sigma$.

\subsection{The unitary dual of $\mathbb{Z}^{d} \rtimes H$ and its Plancherel measure}

Suppose that $A=\mathbb{Z}^{d}=\sum_{k=1}^{d} \mathbb{Z} e_{k}$ is a full rank lattice of $\mathbb{R}^{d}=\sum_{k=1}^{d} \mathbb{R} e_{k}$ and $H$ is a finite subgroup of $G L\left(\mathbb{Z}^{d}\right)$ acting non-trivially on $\mathbb{Z}^{d}$ and let $G=A \rtimes H$ be the semi-direct product group with multiplication law given by $(\ell, M)(\kappa, N)=(\ell+M(\kappa), M N)$.

Since $H$ is assumed to be a finite group, every irreducible representation of the semidirect product group $\mathbb{Z}^{n} \rtimes H$ can be realized as acting in some finite-dimensional Hilbert space. Every unitary irreducible representation of $\mathbb{Z}^{n} \rtimes H$ is obtained by inducing an irreducible representation of a subgroup which is isomorphic to a finite extension of $\mathbb{Z}^{d}$.

Let (see Theorem 7.44 in [2]), $\widehat{G}$ be the collection of equivalent unitary representations (the unitary dual) of $G$. Given an integrable sequence $a$, the group Fourier transform of $a$ is a measurable field of matrices (matrix-valued function) defined over the unitary dual of $G$ as follows. For a unitary irreducible representation $\pi \in \widehat{G}$, the group Fourier transform of $a$ takes the form

$$
\widehat{a}(\pi)=\sum_{(\kappa, M) \in A \rtimes H} a(\kappa, M) \pi\left((\kappa, M)^{-1}\right)=\sum_{(\kappa, M) \in A \rtimes H} a(\kappa, M) \pi\left(\left(-M^{-1} \kappa, M^{-1}\right)\right)
$$

and we may regard $\widehat{a}(\pi)$ as an element of $\mathcal{B}\left(\mathbf{H}_{\pi}\right)$. Indeed, the operator (2.2) is bounded since for any vector $b \in \mathbf{H}_{\pi}$ of unit norm, Triangle Inequality gives

$$
\|\widehat{a}(\pi) b\|_{\mathbf{H}_{\pi}} \leq \sum_{(\kappa, M) \in A \rtimes H}|a(\kappa, M)| \cdot\left\|\pi\left((\kappa, M)^{-1}\right) b\right\|_{\mathbf{H}_{\pi}}=\sum_{(\kappa, M) \in A \rtimes H}|a(\kappa, M)| .
$$

Next, there exists a measure $d \mu$ defined on the unitary dual of $G$ such that the Fourier transform $a \mapsto \widehat{a}$ is an isometric embedding of $l^{1}(A \rtimes H) \cap l^{2}(A \rtimes H)$ into the Hilbert space $\mathbf{B}$ consisting of vectors

$$
f: \widehat{G} \rightarrow \bigcup_{\pi \in \widehat{G}} \mathcal{B}\left(\mathbf{H}_{\pi}\right)
$$

2. See also the discussion above on how to realize each irreducible representation by inducing an irreducible representation of a suitable closed subgroup 
such that (a) $f(\pi) \in \mathcal{B}\left(\mathbf{H}_{\pi}\right)$, (b) $f$ is a measurable field of operators and (c) $\int_{\widehat{G}}\|f(\pi)\|_{\mathrm{HS}}^{2} d \mu(\pi)$ is convergent.

The isometry $a \mapsto \widehat{a}$ extends to a unitary map from $l^{2}(A \rtimes H)$ onto B. For $a, b \in l^{1}(A \rtimes H) \cap$ $l^{2}(A \rtimes H)$, the Plancherel formula for $A \rtimes H$ is given by

$$
\sum_{(\kappa, M) \in A \rtimes H} a((\kappa, M)) \overline{b((\kappa, M))}=\int_{\widehat{G}} \operatorname{Tr}\left(\widehat{a}(\pi)[\widehat{b}(\pi)]^{\star}\right) d \mu(\pi)
$$

and the measure $d \mu$ in (2.3) is called Plancherel measure for $G$.

Note that for $a, b \in l^{1}(A \rtimes H) \cap l^{2}(A \rtimes H)$,

$$
\operatorname{Tr}\left(\widehat{a}(\pi)[\widehat{b}(\pi)]^{\star}\right)=\sum_{k=1}^{\infty}\left\langle\widehat{a}(\pi)[\widehat{b}(\pi)]^{\star} e_{k}, e_{k}\right\rangle_{\mathbf{H}_{\pi}}
$$

where $\left(e_{k}\right)_{k=1}^{\infty}$ is some fixed orthonormal basis of $\mathbf{H}_{\pi}$. Since $a, b$ are assumed to be summable, the trace of the operator $\widehat{a}(\pi) \circ[\widehat{b}(\pi)]^{\star}$ is given by the following summation

$$
\begin{aligned}
\operatorname{Tr}\left(\widehat{a}(\pi) \circ[\widehat{b}(\pi)]^{\star}\right) & =\sum_{\gamma \in A \rtimes H} a(\gamma) \cdot\left\langle\pi(\gamma)[\widehat{b}(\pi)]^{\star} e_{k}, e_{k}\right\rangle_{\mathbf{H}_{\pi}} \\
& =\sum_{\gamma \in A \rtimes H} a(\gamma) \cdot\left\langle e_{k}, \widehat{b}(\pi) \pi\left(\gamma^{-1}\right) e_{k}\right\rangle_{\mathbf{H}_{\pi}} \\
& =\sum_{\gamma \in A \rtimes H} a(\gamma) \cdot \overline{\left\langle\widehat{b}(\pi) \pi\left(\gamma^{-1}\right) e_{k}, e_{k}\right\rangle_{\mathbf{H}_{\pi}}} \\
& =\sum_{\gamma \in A \rtimes H} \sum_{\sigma \in A \rtimes H} a(\gamma) \overline{b(\sigma)} \cdot\left\langle e_{k}, \pi\left(\sigma \gamma^{-1}\right) e_{k}\right\rangle_{\mathbf{H}_{\pi}}
\end{aligned}
$$

and as result,

$$
\left|\operatorname{Tr}\left(\widehat{a}(\pi) \circ[\widehat{b}(\pi)]^{\star}\right)\right| \leq \sum_{\gamma \in A \rtimes H} \sum_{\sigma \in A \rtimes H}|a(\gamma) \cdot b(\sigma)|<\infty .
$$

Next, letting $\delta_{\left(0, \mathrm{id}_{H}\right)}$ be the indicator function of the identity element in $A \rtimes H$, it follows that $h=$ $\delta_{\left(0, \mathrm{id}_{H}\right)} * h$. Defining $h^{*}(x)=\overline{h\left(x^{-1}\right)}$, we obtain

$$
h\left(\left(0, \operatorname{id}_{H}\right)\right)=\left(\delta_{\left(0, \operatorname{id}_{H}\right)} * h\right)\left(\left(0, \operatorname{id}_{H}\right)\right)=\left\langle\delta_{\left(0, \operatorname{id}_{H}\right)}, L\left(\left(0, \operatorname{id}_{H}\right)\right) h^{*}\right\rangle_{l^{2}(A \rtimes H)} .
$$

Applying Formula (2.3), we obtain

$$
\begin{aligned}
h\left(\left(0, \operatorname{id}_{H}\right)\right) & =\left\langle\delta_{\left(0, \mathrm{id}_{H}\right)}, h^{*}\right\rangle_{A \rtimes H} \\
& =\int_{\widehat{G}} \operatorname{Tr}\left(\widehat{\delta_{\left(0, \mathrm{id}_{H}\right)}}(\pi) \circ \widehat{h}(\pi)\right) d \mu(\pi) .
\end{aligned}
$$

Since $\widehat{\delta_{\left(0, \mathrm{id}_{H}\right)}}(\pi)$ is the identity operator,

$$
h\left(\left(0, \operatorname{id}_{H}\right)\right)=\int_{\widehat{G}} \operatorname{Tr}(\widehat{h}(\pi)) d \mu(\pi) .
$$


Let $\chi_{\lambda}$ be a unitary character of $A \times\left\{\operatorname{id}_{H}\right\}$ defined by $\chi_{\lambda}\left(j, \operatorname{id}_{H}\right)=e^{2 \pi i\langle\lambda, j\rangle}$. Given $(k, M) \in G$ and $\left(j, \operatorname{id}_{H}\right) \in A \times\left\{\operatorname{id}_{H}\right\}$, it is clear that

$$
\chi_{\lambda}\left((k, M)^{-1}\left(j, \operatorname{id}_{H}\right)(k, M)\right)=e^{2 \pi i\left\langle\left(M^{-1}\right)^{T} \lambda, j\right\rangle}=\chi_{\left(M^{-1}\right)^{T} \lambda}\left(j, \operatorname{id}_{H}\right) .
$$

Thus, the conjugation action of $G$ on its normal subgroup $A \times\left\{\operatorname{id}_{H}\right\}$ induces a natural action of the group on the unitary dual of $A \times\left\{\operatorname{id}_{H}\right\}$ which we shall identify with the torus $\widehat{A} \simeq \mathbb{R}^{d} / \mathbb{Z}^{d}$. This action is called the dual action of $G$ on $\widehat{A}$ and is described as follows. Given $((k, M), \lambda) \in G \times \widehat{A}$,

$$
((k, M), \lambda) \mapsto\left(M^{-1}\right)^{T} \lambda=(k, M) \star \lambda .
$$

Next, for each $\lambda \in \widehat{A}$, we let

$$
G_{\lambda}=\left\{(k, M) \in A \rtimes H: \chi_{\left(M^{-1}\right)^{T} \lambda}=\chi_{\lambda}\right\}
$$

be the stabilizer of the unitary character $\chi_{\lambda}$. This group plays a central role in the realization of the unitary irreducible representation corresponding to $\lambda$. Referring to Section 6.6 in [2], since the dual orbits in $\widehat{A}$ are finite sets, the map $\beta: G / G_{\lambda} \rightarrow \beta\left(G / G_{\lambda}\right) \subseteq \widehat{A}$ defined explicitly by $\beta\left((k, M) G_{\lambda}\right)=\left(M^{-1}\right)^{T} \lambda$ takes a finite set into another finite set. As a result (see Page 196 of [2]) there exists a Borel set $\Sigma$ in $\widehat{A}$ which intersects each dual orbit in $\widehat{A}$ in exactly one point.

For each $\lambda \in \Sigma$, the subgroup

$$
H_{\lambda}=\left\{(0, M) \in\{0\} \times H: \chi_{\left(M^{-1}\right)^{T} \lambda}=\chi_{\lambda}\right\}
$$

is called the little group. Next, the unitary dual of $G$ is constructed as follows. Firstly, we fix a Borel cross-section (or transversal) $\Sigma \subseteq \widehat{A}$ for the $G$-orbits in the torus. Secondly, for a fixed $\sigma \in \widehat{H_{\lambda}}$, we define a unitary representation $\chi_{\lambda} \otimes \sigma$ of $A \rtimes H_{\lambda}$ acting on $\mathbf{H}_{\sigma}$ as follows. Given $(\kappa, M) \in A \rtimes H_{\lambda}$,

$$
\left(\chi_{\lambda} \otimes \sigma\right)(\kappa, M)=\exp (2 \pi i\langle\lambda, \kappa\rangle) \sigma(M) .
$$

To verify that $\chi_{\lambda} \otimes \sigma$ is a unitary character, we proceed as follows:

$$
\begin{aligned}
\left(\chi_{\lambda} \otimes \sigma\right)\left(\left(\kappa_{1}, M_{1}\right)\left(\kappa_{2}, M_{2}\right)\right) & =\left(\chi_{\lambda} \otimes \sigma\right)\left(\kappa_{1}+M_{1} \kappa_{2}, M_{1} M_{2}\right) \\
& =\exp \left(2 \pi i\left\langle\lambda, \kappa_{1}\right\rangle\right) \exp \left(2 \pi i\left\langle M_{1}^{T} \lambda, \kappa_{2}\right\rangle\right) \sigma\left(M_{1} M_{2}\right) \\
& =\exp \left(2 \pi i\left\langle\lambda, \kappa_{1}\right\rangle\right) \exp \left(2 \pi i\left\langle\lambda, \kappa_{2}\right\rangle\right) \sigma\left(M_{1} M_{2}\right) \\
& =\left(\chi_{\lambda} \otimes \sigma\right)\left(\kappa_{1}, M_{1}\right)\left(\chi_{\lambda} \otimes \sigma\right)\left(\kappa_{2}, M_{2}\right) .
\end{aligned}
$$

A direct application of a result of Lipsman (see [4], Page 76), gives the following result.

The unitary dual of $G$ is parametrized by the fiber set

$$
\widehat{G}=\bigcup_{\lambda \in \Sigma}\left\{\pi_{\lambda, \sigma}=\operatorname{ind}_{A \rtimes H_{\lambda}}^{A \rtimes \chi_{\lambda}}\left(\chi_{\lambda} \otimes \sigma\right): \sigma \in \widehat{H_{\lambda}}\right\}
$$

with base space $\Sigma$.

For each $\lambda$ in the base space, the corresponding fiber can be identified with the unitary dual of $H_{\lambda}$. Each unitary representation $\pi_{\lambda, \sigma}$ acts by left translations on the Hilbert space $l^{2}\left(H / H_{\lambda}, \mathbf{H}_{\sigma}\right)$ which we naturally identify with space of functions $f: G \rightarrow \mathbf{H}_{\sigma}$ such that

$$
f(x y)=\left[\chi_{\lambda} \otimes \sigma\right]\left(y^{-1}\right) f(x)
$$


for all $y \in G_{\lambda}$ and $\sum_{x G_{\lambda} \in G / G_{\lambda}}\|f(x)\|_{\mathbf{H}_{\sigma}}^{2}<\infty$. The inner product on the Hilbert space $l^{2}\left(H / H_{\lambda}, \mathbf{H}_{\sigma}\right)$ is given by

$$
\langle f, g\rangle_{l^{2}\left(H / H_{\lambda}, \mathbf{H}_{\sigma}\right)}=\sum_{x G_{\lambda} \in G / G_{\lambda}}\langle f(x), g(x)\rangle_{\mathbf{H}_{\sigma}} \text { for } f, g \in l^{2}\left(H / H_{\lambda}, \mathbf{H}_{\sigma}\right) .
$$

Note that the unitary dual of $A$ is the $d$-dimensional torus, which we denote by $\mathbb{T}^{d}=[0,1)^{d}$. Next, let $\Sigma \subset[0,1)^{d}$ be a Borel transversal for the $H$-orbits in $[0,1)^{d}$.

Lemma 3. For almost every $\lambda \in \Sigma$, the little group corresponding to $\lambda$ is trivial.

Proof. Let $\mathcal{C}=\{K: K$ is a subgroup of $H$ and $K \neq\{e\}\}=\left\{C_{1}, \cdots, C_{u}\right\}$ be a collection of all subgroups of $H$ other than the identity subgroup. Since $H$ is finite, then $\mathcal{C}$ must be finite as well. Next, for each $k \in\{1, \cdots, u\}$, we define $\Sigma_{k}=\left\{\lambda \in \Sigma: H_{\lambda}=C_{k}\right\}$. In other words, each $\Sigma_{k}$ contains points in $\Sigma$ which are stabilized by the subgroup $C_{k}$. Fixing $k \in\{1, \cdots, u\}$, let $C_{k}^{T}=\left\{B^{T}: B \in C_{k}\right\}=$ $\left\{M_{k_{1}}, \cdots, M_{k_{\ell}}\right\}$. Furthermore, defining $T_{j}=M_{j}-\mathrm{Id}$, and setting

$$
\Sigma_{k}^{(j)}=\left\{\lambda \in \Sigma_{k}: T_{k_{j}}(\lambda) \in \mathbb{Z}^{d}\right\}
$$

we obtain that $\Sigma_{k}=\bigcap_{j=1}^{\ell} \Sigma_{k}^{(j)}$ and $\Sigma=\bigcup_{k=1}^{u} \Sigma_{k} \cup\left\{\lambda \in \Sigma: H_{\lambda}=\{e\}\right\}$. For each fixed $k$,

$$
\Sigma_{k}=\bigcap_{j=1}^{\ell}\left\{\lambda \in \Sigma_{k}: T_{k_{j}}(\lambda) \in \mathbb{Z}^{d}\right\} \subseteq \bigcap_{j=1}^{\ell} T_{k_{j}}(\Sigma) \cap \mathbb{Z}^{d}
$$

and since $T_{k_{j}}(\Sigma)$ is relatively compact, for each fixed $k$, there is a finite set of polynomials $S_{k}=$ $\left\{p_{k_{1}}, \cdots, p_{k_{l}}\right\}$ such that

$$
\Sigma_{k}=\left\{\lambda \in \Sigma: p(\lambda)=0 \text { for all } p \in S_{k}\right\} .
$$

This shows that every $\Sigma_{k}$ has Lebesgue measure zero in $\Sigma$.

Lemma 4. The unitary dual of $G=\mathbb{Z}^{d} \rtimes H$ is parametrized by $\Sigma$ and the Plancherel measure is equal to the usual Lebesgue measure on the torus restricted to $\Sigma$. Moreover, for each $\lambda \in \Sigma$, we may realize the corresponding irreducible representation $\pi_{\lambda}$ as acting in $l^{2}(H)$ as follows. Given $f \in l^{2}(H)$,

$$
\left[\pi_{\lambda}(p) f\right](M)=\left\{\begin{array}{c}
f\left(N^{-1} M\right) \text { if } p=(0, N) \\
e^{2 \pi i\left\langle M^{-1 T} \lambda, k\right\rangle} f(M) \text { if } p=(k, e)
\end{array} .\right.
$$

Proof. We already know that if the little group $H_{\lambda}$ is trivial for almost every $\lambda \in \mathbb{T}^{d}$ then the unitary dual of $G$ is up to a null set with respect to the Lebesgue measure on the $d$-dimensional torus parametrized by $($ see $(2.5))$

$$
\widehat{G}=\left\{\pi_{\lambda}=\operatorname{ind}_{A \rtimes\left\{\operatorname{id}_{H}\right\}}^{G}\left(\chi_{(\lambda, 0)}\right): \lambda \in \Sigma\right\} .
$$

Indeed, we have previously observed that the unitary dual of $G$ is parametrized by the fiber set

$$
\widehat{G}=\bigcup_{\lambda \in \Sigma}\left\{\pi_{\lambda, \sigma}=\operatorname{ind}_{A \rtimes H_{\lambda}}^{A \rtimes H}\left(\chi_{(\lambda, 0)} \otimes \sigma\right): \sigma \in \widehat{H_{\lambda}}\right\}
$$


Since $H_{\lambda}$ is trivial, then its unitary dual is trivial. As a result, we obtain (2.7). In other words, there is a one-to-one correspondence between the unitary dual of $G$ and the cross-section $\Sigma$. Given $f: G \rightarrow \mathbb{C}$ satisfying the additional condition

$$
f(x y)=\chi_{(\lambda, 0)}\left(y^{-1}\right) \cdot f(x) \text { when } y \in \mathbb{Z}^{d} \rtimes\left\{\operatorname{id}_{H}\right\}
$$

we note that

$$
\left[\pi_{\lambda}(0, N) f\right](0, M)=f\left((0, N)^{-1}(0, M)\right)=f\left(0, N^{-1} M\right)
$$

and

$$
\begin{aligned}
{\left[\pi_{\lambda}\left(k, \operatorname{id}_{H}\right) f\right](0, M) } & =f\left(\left(k, \operatorname{id}_{H}\right)^{-1}(0, M)\right) \\
& =f\left((0, M)(0, M)^{-1}\left(k, \operatorname{id}_{H}\right)^{-1}(0, M)\right) \\
& =\chi_{(\lambda, 0)}\left(-M^{-1} k, \operatorname{id}_{H}\right)^{-1} f(0, M) .
\end{aligned}
$$

Next, since $\left(M^{-1} k, \operatorname{id}_{H}\right) \in A \rtimes\left\{\operatorname{id}_{H}\right\}$, we have

$$
\left[\pi_{\lambda}\left(k, \operatorname{id}_{H}\right) f\right](0, M)=\chi_{(\lambda, 0)}\left(M^{-1} k, \operatorname{id}_{H}\right) f(0, M) .
$$

Therefore, each irreducible representation of $G$ is modeled as acting on $l^{2}(H)=\mathbb{C}^{|H|}$ as follows. Given $f \in l^{2}(H)$, we describe the action of $\pi_{\lambda}$ on $f$ as follows

$$
\left[\pi_{\lambda}(p) f\right](M)=\left\{\begin{array}{c}
f\left(N^{-1} M\right) \text { if } p=(0, N) \\
e^{2 \pi i\left\langle M^{-1 T} \lambda, k\right\rangle} f(M) \text { if } p=(k, e)
\end{array} .\right.
$$

Next, given $\phi \in l^{2}(H)=\mathbb{C}^{|H|}, \widehat{a}\left(\pi_{\lambda}\right)$ acts on $\phi$ as follows:

$$
\begin{aligned}
{\left[\widehat{a}\left(\pi_{\lambda}\right) \phi\right](M) } & =\sum_{k \in A} \sum_{N \in H} a(k, N) \pi_{\lambda}\left((k, N)^{-1}\right) \phi(M) \\
& =\sum_{k \in A} \sum_{N \in H} a\left(-N^{-1} k, N^{-1}\right) \exp \left(2 \pi i\left\langle M^{-1 T} \lambda,-N^{-1} k\right\rangle\right) \phi(N M) .
\end{aligned}
$$

Letting $N_{1}=N^{-1} M$, it follows that $N^{-1}=N_{1} M^{-1}$. Thus,

$$
\left[\widehat{a}\left(\pi_{\lambda}\right) \phi\right](M)=\sum_{N_{1} \in H}\left(\sum_{k \in A} a\left(-N_{1} M^{-1} k, N_{1} M^{-1}\right) \exp \left(2 \pi i\left\langle M^{-1 T} \lambda,-N_{1} M^{-1} k\right\rangle\right)\right) \phi\left(N_{1}\right) .
$$

In other words, $\widehat{a}\left(\pi_{\lambda}\right)$ is an integral operator $\left(\widehat{a}\left(\pi_{\lambda}\right)\right.$ is just a matrix) on $l^{2}(M)$ with kernel

$$
K_{a}\left(N_{1}, M\right)=\sum_{k \in A} a\left(-N_{1} M^{-1} k, N_{1} M^{-1}\right) \exp \left(2 \pi i\left\langle M^{-1 T} \lambda,-N_{1} M^{-1} k\right\rangle\right) .
$$

As a result, the square of the Hilbert-Schmidt norm of $\widehat{a}\left(\pi_{\lambda}\right)$ is

$$
\begin{aligned}
\left\|\widehat{a}\left(\pi_{\lambda}\right)\right\|_{\mathrm{HS}}^{2} & =\sum_{M \in H} \sum_{N_{1} \in H}\left|K_{a}\left(N_{1}, M\right)\right|^{2} \\
& =\sum_{M \in H} \sum_{N_{1} \in H}\left|\sum_{k \in \mathbb{Z}^{d}} a\left(-N_{1} M^{-1} k, N_{1} M^{-1}\right) \exp \left(2 \pi i\left\langle M^{-1 T} \lambda,-N_{1} M^{-1} k\right\rangle\right)\right|^{2} .
\end{aligned}
$$


Making the change of variable $\ell=-N_{1} M^{-1} k$, and letting $\mathcal{F}_{1}$ be the partial Fourier transform with respect to the first variable, we obtain

$$
\begin{aligned}
\left\|\widehat{a}\left(\pi_{\lambda}\right)\right\|_{\mathrm{HS}}^{2} & =\sum_{M \in H} \sum_{N_{1} \in H}\left|\sum_{k \in \mathbb{Z}^{d}} a\left(\ell, N_{1} M^{-1}\right) \exp \left(2 \pi i\left\langle M^{-1 T} \lambda, \ell\right\rangle\right)\right|^{2} \\
& =\sum_{M \in H} \sum_{N \in H}\left|\mathcal{F}_{1} a\left(M^{-1 T} \lambda, N_{1} M^{-1}\right)\right|^{2}
\end{aligned}
$$

Next the change of variable $N \mapsto N_{1} M^{-1}$ gives

$$
\left\|\widehat{a}\left(\pi_{\lambda}\right)\right\|_{\mathrm{HS}}^{2}=\sum_{M \in H} \sum_{N \in H}\left|\mathcal{F}_{1} a\left(M^{-1 T} \lambda, N\right)\right|^{2} .
$$

Integrating $\lambda \mapsto\left\|\widehat{a}\left(\pi_{\lambda}\right)\right\|_{\mathrm{HS}}^{2}$ over the cross-section $\Sigma$ with respect to the Lebesgue measure gives

$$
\begin{aligned}
\int_{\Sigma}\left\|\widehat{a}\left(\pi_{\lambda}\right)\right\|_{\mathrm{HS}}^{2} d \lambda & =\int_{\Sigma} \sum_{M \in H} \sum_{N \in H}\left|\mathcal{F}_{1} a\left(M^{-1 T} \lambda, N\right)\right|^{2} d \lambda \\
& =\sum_{N \in H} \int_{\Sigma}\left(\sum_{M \in H}\left|\mathcal{F}_{1} a\left(M^{-1 T} \lambda, N\right)\right|^{2}\right) d \lambda \\
& =\sum_{N \in H_{\mathbb{T}^{d}}} \int_{\left|\mathcal{F}_{1} a(t, N)\right|^{2} d t} \\
& =\sum_{N \in H} \sum_{k \in A}|a(k, N)|^{2} \\
& =\|a\|_{l^{2}(G)}^{2} .
\end{aligned}
$$

Remark 5. Appealing to the Fourier inversion formula as described in (2.4) together with Lemma 4, we obtain that for any suitably chosen sequence a defined on $G$,

$$
a\left(0, \operatorname{id}_{H}\right)=\int_{\Sigma} \operatorname{Tr}\left(\widehat{a}\left(\pi_{\lambda}\right)\right) d \lambda .
$$

Example 6. Let $G=\mathbb{Z} \rtimes \mathbb{Z}_{2}$ be the semidirect product group endowed with the group operation given by $(k, j)\left(k_{1}, j_{1}\right)=\left(k+(-1)^{j} k_{1}, j+j_{1}\right)$. Appealing to the theory of Mackey, the unitary dual of $G$ is up to a null set parametrized by the set $\left(0, \frac{1}{2}\right)$. Moreover, each representation $\pi_{\lambda}$ is realized as acting on the Hilbert space $\mathbb{C}^{2}$ as follows. As such, there exists a basis for $\mathbb{C}^{2}$ with respect to which

$$
\pi_{\lambda}(0,1)=\left[\begin{array}{ll}
0 & 1 \\
1 & 0
\end{array}\right] \text { and } \pi_{\lambda}(1,0)=\left[\begin{array}{cc}
e^{2 \pi i \lambda} & 0 \\
0 & e^{-2 \pi i \lambda}
\end{array}\right] .
$$

Let $a \in l^{2}(G)$ be the uniform distribution on $\{(1,0),(-1,0),(0,1)\}$. In other words, $a=\frac{1}{3}\left(\delta_{(1,0)}+\delta_{(-1,0)}+c\right.$ We will show that for any natural number $n, a^{(2 n)}(0,0)=\frac{1}{9^{n}} \sum_{j=0}^{n}\left(\begin{array}{c}2 n \\ 2 j\end{array}\right) \cdot\left(\frac{(2 j) !}{j !^{2}}\right)$. First, we compute the Fourier transform of a as follows. Given $\lambda \in[0,1)$, we have

$$
\widehat{a}\left(\pi_{\lambda}\right)=\frac{1}{3} \pi_{\lambda}(0,1)+\frac{1}{3} \pi_{\lambda}(1,0)+\frac{1}{3} \pi_{\lambda}(-1,0)=\left[\begin{array}{cc}
\frac{2}{3} \cos (2 \pi \lambda) & \frac{1}{3} \\
\frac{1}{3} & \frac{2}{3} \cos (2 \pi \lambda)
\end{array}\right] .
$$


Secondly, the convolution of a with itself $2 n$ times evaluated at the identity element of $G$ is given by the following calculations:

$$
\begin{aligned}
a^{(2 n)}(0,0) & =\int_{\Sigma} \operatorname{Tr}\left(\widehat{a^{(2 n)}}\left(\pi_{\lambda}\right)\right) d \lambda=\int_{\Sigma} \operatorname{Tr}\left(\widehat{a}\left(\pi_{\lambda}\right)^{2 n}\right) d \lambda \\
& =\int_{\left(0, \frac{1}{2}\right)} \operatorname{Tr}\left(\left[\begin{array}{cc}
\frac{2}{3} \cos (2 \pi \lambda) & \frac{1}{3} \\
\frac{1}{3} & \frac{2}{3} \cos (2 \pi \lambda)
\end{array}\right]^{2 n}\right) d \lambda .
\end{aligned}
$$

To obtain a satisfactory formula for $a^{(2 n)}(0,0)$, we will need to compute the trace of the matrix appearing in the integral above. Since the eigenvalues of a $\left(\pi_{\lambda}\right)$ are given by $\frac{2}{3} \cos (2 \pi \lambda)-\frac{1}{3}$ and $\frac{2}{3} \cos (2 \pi \lambda)+\frac{1}{3}$, we obtain

$$
\operatorname{Tr}\left(\left[\begin{array}{cc}
\frac{2}{3} \cos (2 \pi \lambda) & \frac{1}{3} \\
\frac{1}{3} & \frac{2}{3} \cos (2 \pi \lambda)
\end{array}\right]^{2 n}\right)=\left(\frac{2}{3} \cos 2 \pi \lambda-\frac{1}{3}\right)^{2 n}+\left(\frac{2}{3} \cos 2 \pi \lambda+\frac{1}{3}\right)^{2 n}
$$

and consequently,

$$
\begin{aligned}
a^{(2 n)}(0,0) & =\int_{\left(0, \frac{1}{2}\right)}\left(\left(\frac{2}{3} \cos 2 \pi \lambda-\frac{1}{3}\right)^{2 n}+\left(\frac{2}{3} \cos 2 \pi \lambda+\frac{1}{3}\right)^{2 n}\right) d \lambda \\
& =\frac{1}{2} \int_{(0,1)}\left(\left(\frac{2}{3} \cos 2 \pi \lambda-\frac{1}{3}\right)^{2 n}+\left(\frac{2}{3} \cos 2 \pi \lambda+\frac{1}{3}\right)^{2 n}\right) d \lambda .
\end{aligned}
$$

Moving forward, let $\Gamma$ be the Gamma function defined as

$$
\Gamma(z)=\int_{0}^{\infty} x^{z-1} e^{-x} \text { for } z \in \mathbb{C} \text { and } \operatorname{Re}(z)>0 .
$$

Straightforward calculations yield the following. For fixed $\iota \in\{1,-1\}$

$$
\begin{aligned}
& \frac{1}{2} \int_{(0,1)} \sum_{k=0}^{2 n}\left(\begin{array}{c}
2 n \\
k
\end{array}\right)\left(\frac{2}{3} \cos 2 \pi \lambda\right)^{k} \cdot\left(\frac{\iota}{3}\right)^{2 n-k} d \lambda \\
& =\frac{1}{2} \sum_{k=0}^{2 n}\left(\begin{array}{c}
2 n \\
k
\end{array}\right) \int_{(0,1)}\left(\frac{2}{3} \cos 2 \pi \lambda\right)^{k} \cdot\left(\frac{\iota}{3}\right)^{2 n-k} d \lambda \\
& =\frac{1}{2} \sum_{j=0}^{2 n}\left(\begin{array}{c}
2 n \\
2 j
\end{array}\right) \cdot\left(\frac{2^{2 j-2} \times 9^{-n} \times 4 \times \Gamma\left(j+\frac{1}{2}\right)}{\sqrt{\pi} \times \Gamma(1+j)}+\frac{2^{2 j-2} \times 9^{-n} \times 4 \times \Gamma\left(j+\frac{1}{2}\right)}{\sqrt{\pi} \times \Gamma(1+j)}\right) .
\end{aligned}
$$

Since $\Gamma(1+j)=j !$ and $\Gamma\left(j+\frac{1}{2}\right)=\frac{(2 j) !}{4^{j} j !} \sqrt{\pi}$, it follows that

$$
\begin{aligned}
a^{(2 n)}(0,0) & =\sum_{j=0}^{2 n}\left(\begin{array}{c}
2 n \\
2 j
\end{array}\right) \cdot\left(\frac{1}{2}\left(\frac{2^{2 j-2} \times 9^{-n} \times 4}{j !} \frac{(2 j) !}{4^{j} j !}+\frac{2^{2 j-2} \times 9^{-n} \times 4}{j !} \frac{(2 j) !}{4^{j} j !}\right)\right) \\
& =\frac{1}{9^{n}} \sum_{j=0}^{2 n}\left(\begin{array}{c}
2 n \\
2 j
\end{array}\right) \cdot\left(\frac{(2 j) !}{j !^{2}}\right)=\frac{1}{9^{n}} \sum_{j=0}^{2 n}\left(\begin{array}{c}
2 n \\
2 j
\end{array}\right) \cdot\left(\begin{array}{c}
2 j \\
j
\end{array}\right) .
\end{aligned}
$$

We are now ready to prove our main results. 


\subsection{Proof of Theorem 1}

Let

$$
K=\left\{\left( \pm e_{1}, \operatorname{id}_{H}\right), \cdots,\left( \pm e_{d}, \operatorname{id}_{H}\right),\left(0, h_{i_{1}}\right), \cdots,\left(0, h_{i_{m}}\right)\right\}
$$

be a minimal symmetric generating set for $G$. Then $|K|=2 d+m$. Next, let $a$ be a sequence of finite support defined on $G$ such that

$$
a(x)=\left\{\begin{array}{c}
\frac{1}{2 d+m} \text { if } x \in K \\
0 \text { otherwise }
\end{array}\right.
$$

The group Fourier transform of $a$ takes the form

$$
\begin{aligned}
& \widehat{a}\left(\pi_{\lambda}\right)=|K|^{-1} \overbrace{\sum_{k=1}^{d}\left(\sum_{s=0}^{1} \operatorname{ind}_{\mathbb{Z}^{d} \rtimes\left\{\operatorname{id}_{H}\right\}}^{G}\left(\chi_{(\lambda, 0)}\right)\left((-1)^{s} e_{k}, \mathrm{id}_{H}\right)\right)}^{=\mathbf{A}_{D(\lambda)}} \\
& +|K|^{-1} \underbrace{\sum_{j=1}^{m} \operatorname{ind}_{\mathbb{Z}^{d} \rtimes\left\{\operatorname{id}_{H}\right\}}^{G}\left(\chi_{(\lambda, 0)}\right)\left(0, h_{r_{j}}\right)}_{=\mathbf{A}_{S}} \text {. }
\end{aligned}
$$

Since (see (2.6))

$$
\begin{aligned}
\sum_{k=1}^{d} \sum_{s=0}^{1}\left[\operatorname{ind}_{\mathbb{Z}^{d} \rtimes\left\{\operatorname{id}_{H}\right\}}^{G}\left(\chi_{(\lambda, 0)}\right)\left((-1)^{s} e_{k}, \operatorname{id}_{H}\right) f\right](h) & =\sum_{k=1}^{d} \sum_{s=0}^{1} e^{2 \pi i\left\langle\left(h^{-1}\right)^{T} \lambda,(-1)^{s} e_{k}\right\rangle} f(h) \\
& =\sum_{k=1}^{d} \sum_{s=0}^{1} e^{2 \pi i\left\langle\lambda,(-1)^{s} h^{-1} e_{k}\right\rangle} f(h) \\
& =2 \sum_{k=1}^{d} \cos \left(2 \pi\left\langle\lambda, h^{-1} e_{k}\right\rangle\right) f(h)
\end{aligned}
$$

there exists a basis for $l^{2}(H)$ such that the matrix representation of $\mathbf{A}_{D(\lambda)}$ is given by the real diagonal matrix

$$
D(\lambda)=\left[\begin{array}{ccc}
2 \sum_{k=1}^{d} \cos \left(2 \pi\left\langle\lambda, h_{1}^{-1} e_{k}\right\rangle\right) & \\
& \ddots & \\
& & 2 \sum_{k=1}^{d} \cos \left(2 \pi\left\langle\lambda, h_{|H|}^{-1} e_{k}\right\rangle\right)
\end{array}\right]
$$

Next, let $S$ be the matrix representation of the linear operator $\mathbf{A}_{S}$ which is an element of the algebra generated by the left regular representation of $H$. Then

$$
a_{K}^{(2 n)}\left(0, \mathrm{id}_{H}\right)=\int_{\Sigma} \operatorname{Tr}\left(\left(\frac{D(\lambda)+S}{|K|}\right)^{2 n}\right) d \lambda=\frac{1}{|H|} \int_{[0,1]^{d}} \operatorname{Tr}\left(\left(\frac{D(\lambda)+S}{|K|}\right)^{2 n}\right) d \lambda
$$


Assuming that $D(\lambda)$ and $S$ (as defined in (1.2) and (1.3) respectively) are simultaneously triangularizable for almost every $\lambda$ with respect to the Lebesgue measure in $[0,1]^{d}$, there exists an invertible matrix $P$ such that $P D(\lambda) P^{-1}$ and $P S P^{-1}$ are upper triangular. We may then write

$$
P(D(\lambda)+S) P^{-1}=P D(\lambda) P^{-1}+P S P^{-1}=\left[\begin{array}{cccc}
d_{1}(\lambda)+s_{1} & * & \cdots & * \\
& & * & \vdots \\
& & * \\
& & d_{|H|}(\lambda)+s_{|H|}
\end{array}\right]
$$

for some complex numbers $d_{j}(\lambda), s_{l}$ and $\operatorname{Tr}\left((D(\lambda)+S)^{2 n}\right)=\sum_{j=1}^{|H|}\left(d_{j}(\lambda)+s_{j}\right)^{2 n}$. Therefore, there exists a sequence $\left(\omega_{s}\right)_{s=1}^{|H|}$ of eigenvalues for $S$ such that

$$
\begin{aligned}
a_{K}^{(2 n)}\left(0, \operatorname{id}_{H}\right) & =\frac{1}{|H|} \int_{[0,1]^{d}} \operatorname{Tr}\left(\left(\frac{D(\lambda)+S}{|K|}\right)^{2 n}\right) d \lambda \\
& =\frac{1}{|H|} \sum_{s=1}^{|H|} \int_{[0,1]^{d}}\left(\left(\sum_{k=1}^{d} \frac{2 \cos \left(2 \pi\left\langle\lambda, h_{s}^{-1} e_{k}\right\rangle\right)}{|K|}\right)+\frac{\omega_{s}}{|K|}\right)^{2 n} d \lambda
\end{aligned}
$$

as desired.

\subsection{Proof of Corollary 2}

Suppose that for almost every $\lambda, D(\lambda)$ is a constant multiple of the identity. Then for each $1 \leq s \leq$ $|H|$,

$$
\sum_{k=1}^{d} \frac{2 \cos \left(2 \pi\left\langle\lambda, h_{s}^{-1} e_{k}\right\rangle\right)}{|K|}=\sum_{k=1}^{d} \frac{2 \cos \left(2 \pi\left\langle\lambda, e_{k}\right\rangle\right)}{|K|}=\sum_{k=1}^{d} \frac{2 \cos \left(2 \pi \lambda_{k}\right)}{|K|}
$$

Next,

$$
\begin{aligned}
a_{K}^{(2 n)}\left(0, \mathrm{id}_{H}\right) & =\frac{1}{|H|} \sum_{s=1}^{|H|} \int_{[0,1]^{d}}\left(\left(\sum_{k=1}^{d} \frac{2 \cos \left(2 \pi \lambda_{k}\right)}{|K|}\right)+\frac{\omega_{s}}{|K|}\right)^{2 n} d \lambda \\
& =\frac{1}{|H| \cdot|K|^{2 n}} \sum_{s=1}^{|H|} \int\left(\left(\sum_{[0,1]^{d}}^{d} 2 \cos \left(2 \pi \lambda_{k}\right)\right)+\omega_{s}\right)^{2 n} d \lambda \\
& =\frac{1}{|H| \cdot|K|^{2 n}} \sum_{s=1}^{|H|} \int \sum_{[0,1]^{d}}^{\ell=0}\left(\begin{array}{c}
2 n \\
\ell
\end{array}\right)\left(\sum_{k=1}^{d} 2 \cos \left(2 \pi \lambda_{k}\right)\right)^{\ell}\left(\omega_{s}\right)^{2 n-\ell} d \lambda \\
& =\frac{1}{|H| \cdot|K|^{2 n}} \sum_{s=1}^{|H|} \sum_{\ell=0}^{2 n}\left(\begin{array}{c}
2 n \\
\ell
\end{array}\right)\left(\omega_{s}\right)^{2 n-\ell} \int_{[0,1]^{d}}\left(\sum_{k=1}^{d} 2 \cos \left(2 \pi \lambda_{k}\right)\right)^{\ell} d \lambda .
\end{aligned}
$$


To simplify the last integral above, we appeal to the Multinomial Theorem, as follows

$$
\begin{aligned}
& \int_{[0,1)^{d}}\left(\sum_{j=1}^{d} 2 \cos \left(2 \pi \lambda_{j}\right)\right)^{\ell} d \lambda \\
= & \int_{[0,1)^{d}} \sum_{k_{1}+\ldots+k_{d}=\ell} \frac{2^{\ell} \ell !}{k_{1} ! \cdots k_{d} !} \prod_{r=1}^{d} \cos ^{k_{r}}\left(2 \pi \lambda_{r}\right) d \lambda \\
= & \int_{0}^{1} \cdots \int_{0}^{1} \sum_{k_{1}+\ldots+k_{d}=\ell} \frac{2^{\ell} \ell !}{k_{1} ! \cdots k_{d} !}\left(\cos ^{k_{1}}\left(2 \pi \lambda_{1}\right) \cdots \cos ^{k_{d}}\left(2 \pi \lambda_{d}\right)\right) d \lambda_{1} \cdots d \lambda_{d} \\
= & \sum_{k_{1}+\ldots+k_{d}=\ell} \frac{2^{\ell} \ell !}{k_{1} ! \cdots k_{d} !}\left(\int_{0}^{1} \cos ^{k_{1}}\left(2 \pi \lambda_{1}\right) d \lambda_{1}\right) \cdots\left(\int_{0}^{1} \cos ^{k_{d}}\left(2 \pi \lambda_{d}\right) d \lambda_{d}\right) \\
= & \sum_{k_{1}+\ldots+k_{d}=\ell} \frac{2^{\ell} \ell !}{k_{1} ! \cdots k_{d} !} \prod_{r=1}^{d} \int_{0}^{1}\left(\cos ^{k_{r}}(2 \pi t)\right) d t .
\end{aligned}
$$

Next, straightforward calculations give

$$
\begin{aligned}
\prod_{r=1}^{d} \int_{0}^{1}(\cos (2 \pi t))^{k_{r}} d t & =\prod_{r=1}^{d} 2^{-k_{r}} \sum_{\vartheta=0}^{k_{r}}\left(\begin{array}{c}
k_{r} \\
\vartheta
\end{array}\right)\left(\int_{0}^{1} e^{i 2 \pi t\left(2 \vartheta-k_{r}\right)} d t\right) \\
& =\prod_{r=1}^{d} 2^{-k_{r}} \sum_{\vartheta=0}^{k_{r}}\left(\begin{array}{c}
k_{r} \\
\vartheta
\end{array}\right) \delta_{2 \vartheta-k_{r}, 0} .
\end{aligned}
$$

Since

$$
\int_{[0,1)^{d}}\left(\sum_{j=1}^{d} 2 \cos \left(2 \pi \lambda_{j}\right)\right)^{\ell} d \lambda=\sum_{k_{1}+\ldots+k_{d}=\ell} \frac{2^{\ell} \ell !}{k_{1} ! \cdots k_{d} !} \prod_{r=1}^{d} 2^{-k_{r}} \sum_{\vartheta=0}^{k_{r}}\left(\begin{array}{c}
k_{r} \\
\vartheta
\end{array}\right) \delta_{2 \vartheta-k_{r}, 0},
$$

coming back to (2.14), we obtain

$$
a_{K}^{(2 n)}\left(0, \operatorname{id}_{H}\right)=\frac{1}{|H| \cdot|K|^{2 n}} \sum_{s=1}^{|H|} \sum_{\ell=0}^{2 n} \sum_{k_{1}+\ldots+k_{d}=\ell} \frac{\left(\begin{array}{c}
2 n \\
\ell
\end{array}\right)\left(\omega_{s}\right)^{2 n-\ell} 2^{\ell} \ell !}{k_{1} ! \cdots k_{d} !} \prod_{r=1}^{d} 2^{-k_{r}} \sum_{\vartheta=0}^{k_{r}}\left(\begin{array}{c}
k_{r} \\
\vartheta
\end{array}\right) \delta_{2 \vartheta-k_{r}, 0} .
$$

Observing that

$$
\delta_{2 \vartheta-k_{r}, 0}=\left\{\begin{array}{l}
0 \text { if } k_{r} \neq 2 \vartheta \\
1 \text { if } k_{r}=2 \vartheta
\end{array}\right.
$$

it follows that

$$
a_{K}^{(2 n)}\left(0, \mathrm{id}_{H}\right)=\frac{1}{|H| \cdot|K|^{2 n}} \sum_{s=1}^{|H|} \sum_{\ell=0}^{2 n} \sum_{\substack{k_{1}+\ldots+k_{d}=\ell \\
k_{r} \text { is vern }}} \frac{\left(\begin{array}{c}
2 n \\
\ell
\end{array}\right)\left(\omega_{s}\right)^{2 n-\ell} 2^{\ell} \ell !}{k_{1} ! \cdots k_{d} !} \prod_{r=1}^{d} 2^{-k_{r}}\left(\begin{array}{c}
k_{r} \\
\frac{k_{r}}{2}
\end{array}\right) .
$$

Now,

$$
\prod_{r=1}^{d} 2^{-k_{r}}\left(\begin{array}{c}
k_{r} \\
\frac{k_{r}}{2}
\end{array}\right)=2^{-k_{1} \cdots-k_{d}} \frac{k_{1} !}{\left(\frac{k_{1}}{2}\right) !^{2}} \cdots \frac{k_{d} !}{\left(\frac{k_{d}}{2}\right) !^{2}}
$$


and as a result,

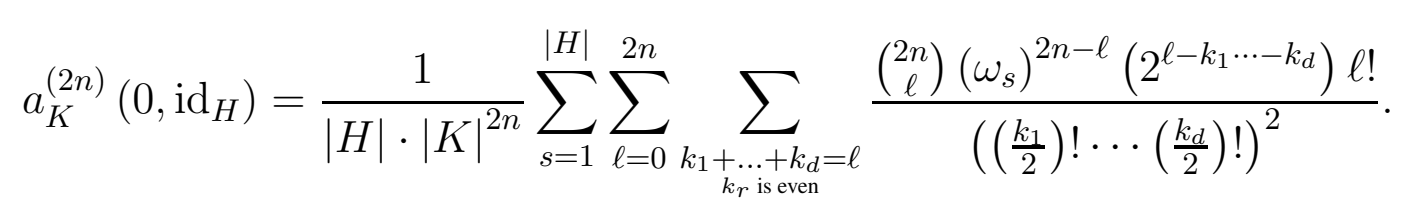

Since $2^{\ell-k_{1} \cdots-k_{d}}=1$, and $\left(\begin{array}{c}2 n \\ \ell\end{array}\right)=\frac{(2 n) !}{\ell !(2 n-\ell) !}$, we obtain

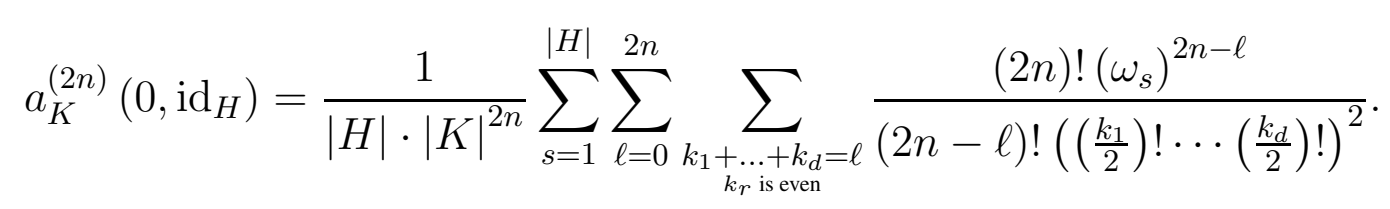

Now, every even index $k_{r}$ takes the form $k_{r}=2 \kappa_{r}$ and

$$
a_{K}^{(2 n)}\left(0, \operatorname{id}_{H}\right)=\frac{1}{|H| \cdot|K|^{2 n}} \sum_{s=1}^{|H|} \sum_{\ell=0}^{2 n} \sum_{2 \kappa_{1}+\ldots+2 \kappa_{d}=\ell} \frac{(2 n) !\left(\omega_{s}\right)^{2 n-\ell}}{(2 n-\ell) !\left(\kappa_{1} ! \cdots\left(\kappa_{d}\right) !\right)^{2}} .
$$

In the above, the condition $2 \kappa_{1}+\ldots+2 \kappa_{d}=\ell$, and by re-indexing, the last summation above,

$$
a_{K}^{(2 n)}\left(0, \operatorname{id}_{H}\right)=\frac{1}{|H| \cdot|K|^{2 n}} \sum_{s=1}^{|H|} \sum_{\ell=0}^{n} \sum_{\kappa_{1}+\ldots+\kappa_{d}=\ell} \frac{(2 n) !\left(\omega_{s}\right)^{2(n-\ell)}}{(2(n-\ell)) !\left(\kappa_{1} ! \cdots\left(\kappa_{d}\right) !\right)^{2}} .
$$

This establishes (1.6) as desired.

\section{The limitations of our representation theoretic approach}

First of all it is worth noting that there are several examples in which the sufficient conditions of Theorem 1 are not met. For instance, let $M=\left[\begin{array}{ll}0 & -1 \\ 1 & -1\end{array}\right]$ and put $G=\mathbb{Z}^{2} \rtimes\langle M\rangle$. For this group extension of $\mathbb{Z}^{2}$, it is easy to verify that $D(\lambda)$ is a diagonal matrix with eigenvalues $2\left(\cos \left(2 \pi \lambda_{1}\right)+\cos \left(2 \pi \lambda_{2}\right)\right)$, $2\left(\cos \left(2 \pi \lambda_{1}\right)+\cos \left(2 \pi\left(\lambda_{1}+\lambda_{2}\right)\right)\right)$ and $2\left(\cos \left(2 \pi \lambda_{2}\right)+\cos \left(2 \pi\left(\lambda_{1}+\lambda_{2}\right)\right)\right)$. Next since $M$ generates a cyclic group of order three, we have (see (1.3))

$$
S=\left[\begin{array}{lll}
0 & 0 & 1 \\
1 & 0 & 0 \\
0 & 1 & 0
\end{array}\right]+\left[\begin{array}{lll}
0 & 0 & 1 \\
1 & 0 & 0 \\
0 & 1 & 0
\end{array}\right]^{-1}=\left[\begin{array}{lll}
0 & 1 & 1 \\
1 & 0 & 1 \\
1 & 1 & 0
\end{array}\right]
$$

It is easy to verify that the eigenvalues of $S$ are given by the sequence $(-1,-1,2)$. However, for almost every $\left(\lambda_{1}, \lambda_{2}\right) \in \mathbb{R}^{2}, S$ and $D(\lambda)$ are not simultaneously triangularizable. If this was the case, then the Lie algebra spanned by $S, D(\lambda)$ would be solvable. However, one can verify that for almost every $\lambda$, the Lie algebra generated by $D(\lambda)$ and $S$ is the algebra of all square matrices of order three (which is not a solvable Lie algebra). Thus, even for such a simple example, there seems to be no simple way to compute the trace of $(D(\lambda)+S)^{n}$ for arbitrary $n$.

Secondly, recall that a unitary representation $\pi$ of a locally compact group $G$ is called primary if the center of its commutant algebra consists only of scalar multiples of the identity. According to Schur's lemma, every irreducible representation of $G$ is a factor representation and the concept of a factor representation can be naturally regarded as an extension of that of irreducibility. Next, we say that $G$ is a type 
I group if every factor representation of $G$ is a direct sum of copies of a unitary irreducible representation of $G$. According to a result of Glimm (Theorem 7.6, [2]) a group is type I if and only if the Mackey Borel structure on its unitary dual is countably separated (see the discussion on Page 205, [2]). In less technical terms, it is virtually impossible to obtain a reasonable parametrization of the unitary dual of a non-type I group. Most infinite non-abelian discrete groups are non type I groups. In fact, a well-known result of Thoma [6] states that ([6]) a discrete group is type I if and only if it possesses an abelian normal subgroup of finite index. For these reasons, we are unable to extend our methods to cases where $G=\mathbb{Z}^{d} \rtimes H$ and $H$ is countably infinite. For instance, the case

$$
G=\left\{\left[\begin{array}{lll}
1 & l & k \\
0 & 1 & j \\
0 & 0 & 1
\end{array}\right]: k, j, l \in \mathbb{Z}\right\}
$$

of the infinitely countable Heisenberg group generated by

$$
\left[\begin{array}{lll}
1 & 0 & 0 \\
0 & 1 & 1 \\
0 & 0 & 1
\end{array}\right],\left[\begin{array}{lll}
1 & 1 & 0 \\
0 & 1 & 0 \\
0 & 0 & 1
\end{array}\right]
$$

cannot be resolved by the methods presented in this work.

\section{References}

[1] Daniel Bump, Persi Diaconis, Angela Hicks, Laurent Miclo, and Harold Widom. An exercise(?) in Fourier analysis on the Heisenberg group. Ann. Fac. Sci. Toulouse Math. (6), 26(2):263-288, 2017. MR3640891.

[2] Gerald B. Folland. A course in abstract harmonic analysis. Textbooks in Mathematics. CRC Press, Boca Raton, FL, second edition, 2016. MR3444405.

[3] Eberhard Kaniuth and Keith F. Taylor. Induced representations of locally compact groups, volume 197 of Cambridge Tracts in Mathematics. Cambridge University Press, Cambridge, 2013. MR3012851.

[4] Ronald L. Lipsman. Group representations. A survey of some current topics. Lecture Notes in Mathematics, Vol. 388. Springer-Verlag, Berlin-New York, 1974. MR0372116.

[5] Ken ichi Tahara. On the finite subgroups of GL(3, Z). Nagoya Math. J., 41:169-209, 1971. MR0272910.

[6] Elmar Thoma. Eine Charakterisierung diskreter Gruppen vom Typ I. Invent. Math., 6:190-196, 1968. MR0248288.

[7] Wolfgang Woess. Random walks on infinite graphs and groups, volume 138 of Cambridge Tracts in Mathematics. Cambridge University Press, Cambridge, 2000. MR1743100. 